\title{
Sinusitis aguda invasiva por Exserohilum rostratum en una paciente pediátrica con recaída medular de leucemia linfoblástica aguda
}

\author{
Invasive acute sinusitis by Exserohilum rostratum in a patient with \\ medullary relapse of acute lymphoblastic leukemia
}

\author{
Dra. Ma. Florencia Astolfo ${ }^{a}$, Dra. Patricia Cañazares ${ }^{a}$,Dra. Elena Majek ${ }^{b}$ Dra. Virginia Burgesserc, \\ Dr. Martín Caruso ${ }^{d}$, Dr. José Basco ${ }^{e}$ Dr. César Alvarado ${ }^{e}$ y Dra. Susana Carnovalef
}

\begin{abstract}
RESUMEN
La infección fúngica invasora ha aumentado en frecuencia a lo largo de la última década, y la sinusitis fúngica es cada vez más habitual.

Los hongos del género Exserohilum (familia Pleosporaceae, orden Pleosporales) son filamentosos y dematiáceos, de localización ubicua. Se trata de patógenos emergentes, que producen, en la mayoría de los casos, infecciones sistémicas que afectan, principalmente, a los senos paranasales y los pulmones. Son más frecuentes en pacientes inmunosuprimidos, aunque pueden presentarse en pacientes inmunocompetentes. El tratamiento de estas infecciones comprende el tratamiento antifúngico, resección quirúrgica y restitución de la inmunidad.

Se presenta el caso de una paciente con recaída medular de leucemia linfoblástica aguda con sinusitis fúngica invasiva por Exserohilum rostratum.

Palabras clave: Exserohilum rostratum, sinusitis, infecciones fúngicas invasoras, leucemia, inmunosupresión.
\end{abstract}

\begin{abstract}
Invasive fungal infection has increased in frequency over the last decade, with fungal sinusitis becoming more frequent.

The fungi of the genus Exserohilum (family Pleosporaceae, order Pleosporales) are filamentous and dematiaceous of ubiquitous location. It is an emerging pathogen, which in most cases produces a systemic infection that mainly affects the paranasal sinuses and lungs. It is more common in immunosuppressed patients, although it may occur in immunocompetent patients. The treatment is based on three pillars: antifungal treatment, surgical debridement and restitution of immunity.
\end{abstract}

a. Departamento de Medicina.

b. Unidad de Oncohematología.

c. Servicio de Anatomía Patológica.

d. Servicio de Infectología.

e. Servicio de Otorrinolaringología.

Hospital Materno Infantil "Dr. Héctor Quintana",

San Salvador de Jujuy, Argentina.

f. Servicio de Micología. Hospital de Pediatría "Prof. Dr.

Juan P. Garrahan", Ciudad de Buenos Aires, Argentina.

Correspondencia:

Dra. Ma. Florencia Astolfo: florencia.astolfo@gmail.com

Financiamiento: Ninguno.

Conflicto de intereses: Ninguno que declarar.

Recibido: 15-8-2017

Aceptado: 14-2-2018
We present the case of a patient with medullary relapse of acute lymphoblastic leukemia with invasive fungal sinusitis by Exserohilum rostratum.

Key words: Exserohilum rostratum, sinusitis, invasive fungal infections, leukemia, immunosuppression.

http: / / dx.doi.org/10.5546/aap.2018.e594

Cómo citar: Astolfo MF, Cañazares P, Majek E. Sinusitis aguda invasiva por Exserohilum rostratum en una paciente pediátrica con recaída medular de leucemia linfoblástica aguda. Arch Argent Pediatr 2018;116(4):e594-e598.

\section{INTRODUCCIÓN}

La infección fúngica invasora (IFI) ha aumentado en frecuencia a lo largo de la última década. Este incremento se debe, por una parte, a la existencia de un mayor número de pacientes sometidos a tratamientos inmunosupresores o a terapias invasivas $\mathrm{y}$, por otra, a los nuevos métodos diagnósticos, microbiológicos y no microbiológicos utilizados. Los agentes etiológicos más frecuentes son Candida albicans y Aspergillus fumigatus. ${ }^{1}$

La sinusitis fúngica, antes considerada un trastorno infrecuente, en la actualidad, es relativamente común y se informa con mayor frecuencia en todo el mundo, aunque, a menudo, se diagnostica de manera errónea. ${ }^{2}$

Los hongos del género Exserohilum (familia Pleosporaceae, orden Pleosporales) son filamentosos y dematiáceos, de localización ubicua, que se encuentran en los suelos y vegetales. Generan enfermedad invasiva y no invasiva tanto en inmunocompetentes como en inmunosuprimidos. ${ }^{3}$

Durante las últimas décadas, aumentó el número de casos reportados de infección por Exserohilum $^{3}$ spp.

A continuación, se presenta el caso de una paciente con recaída medular de leucemia linfoblástica aguda (LLA) con sinusitis fúngica invasiva por Exserohilum rostratum. 


\section{CASO CLÍNICO}

Paciente de sexo femenino, de 3 años y 8 meses de edad, a quien, a los 18 meses de vida, se le diagnosticó LLA precursor B de alto riesgo (por MLL positivo).

Durante la fase de mantenimiento (6-mercaptopurina y metotrexato por vía oral), consultó por fiebre de $38^{\circ} \mathrm{C}$ de 24 h de evolución y tos productiva. Examen físico con regular estado general, hipoventilación en la base pulmonar derecha. Se realizó un hemograma, y el recuento de leucocitos fue $1200 / \mathrm{mm}^{3}$ (blastos: $30 \%$ ); hemoglobina: $8 \mathrm{~g} / \mathrm{dl}$; hematocrito: $23 \%$; recuento de plaquetas: $13000 / \mathrm{mm}^{3}$; radiografía de tórax con infiltrado en la base pulmonar derecha. Se consideró neutropenia febril y, luego de realizar los cultivos correspondientes, se medicó con cefotaxima-amikacina, que cubrió el foco respiratorio. Cumplió 10 días con buena evolución clínica.

Se realizó una punción-aspiración de médula ósea (PAMO) y se confirmó la recaída medular aislada temprana los 22 meses de la remisión completa $(\mathrm{RC})$, por lo que comenzó con quimioterapia según el protocolo GATLA REC 2011. A los 14 días de internación, presentó un edema en la hemicara izquierda y congestión nasal; en el paladar duro, se observó una placa negruzca erosiva de $2 \mathrm{~cm}$ aproximadamente. Se realizó una tomografía axial computada (TAC) de los senos paranasales, con engrosamiento de los senos maxilar y etmoidal izquierdos (Figura 1). Dada la alta presunción de rinosinusitis micótica, comenzó con anfotericina B liposomal a razón de $3 \mathrm{mg} / \mathrm{kg} /$ día. A las 24 h, se realizó una biopsia, que se envió a Anatomía Patológica y a estudio microbiológico al Laboratorio de Micología del Instituto Carlos G. Malbrán. En el examen microscópico directo del material, se informó la presencia de hongos filamentosos. Al mes, se recibió el informe final y se identificó el hongo como Exserohilum sp. Dado que la muestra se encontraba contaminada con ácaros ambientales, no se descartó que el aislamiento pudiera corresponder a una contaminación.

Anatomía Patológica informó extensa angioinvasión por hongos filamentosos y necrosis de la mucosa nasal (Figura 2).

FIgURA 2. Microscopía, angioinvasión: luz vascular ocupada por múltiples estructuras micóticas en forma de esporos e hifas gruesas, tabicadas. Tinción de PAS, $10 \mathrm{X}$

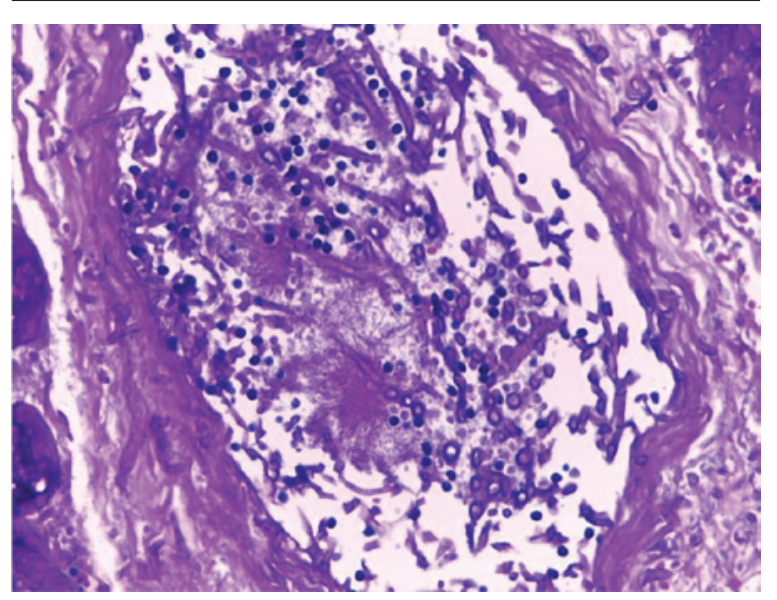

FIGURA 1. Fotografía de la paciente, en la que se observa una placa negruzca erosiva en el paladar duro, y corte coronal de la tomografía axial computada de los senos paranasales, donde se evidencia un engrosamiento de los senos etmoidal y maxilar izquierdos

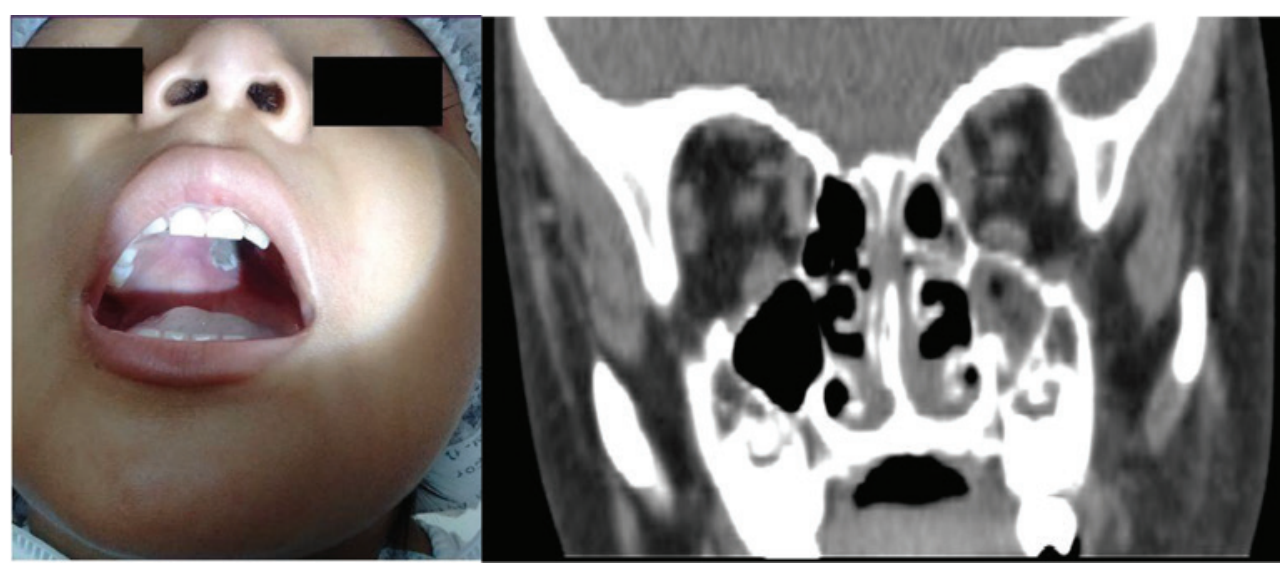


La paciente presentó mejoría clínica y sinusal, persistió con neutropenia prolongada y profunda por tres semanas, con episodios intermitentes de fiebre, y recibió, además del tratamiento antifúngico, múltiples esquemas antibióticos de amplio espectro. Se realizó la búsqueda de focos profundos, incluida la TAC de pulmón, con informe normal. Se aumentó la anfotericina a $5 \mathrm{mg} / \mathrm{kg} /$ día.

Se constató una segunda $\mathrm{RC}$ de la recaída de LLA luego de recibir quimioterapia de inducción, por lo cual recibió 4 días de factor estimulante de colonias, lo que logró la recuperación hematológica.

Se realizó una videoconferencia con el Hospital de Pediatría Juan P. Garrahan (Clínica, Oncología, Infectología y Otorrinolaringología) y se concluyó aumentar la dosis de anfotericina a $7,5 \mathrm{mg} / \mathrm{kg} /$ día, toilette quirúrgica y un nuevo envío de muestras para biopsia, cultivo y biología molecular para el diagnóstico. Se recibió el informe del Laboratorio de Micología del Hospital Garrahan. Se confirmó la observación de gran cantidad de filamentos fúngicos dematiáceos en el material remitido. Esto habría descartado la posibilidad de que el aislamiento del hongo correspondiese a una contaminación. El hongo fue identificado por su aspecto macroscópico y su micromorfología característica en los cultivos (Figura 3). Estos estudios y la confirmación por estudios moleculares permitieron identificar el hongo como Exserohilum rostratum (Figura 3).

Recibió en total 8 semanas de anfotericina B liposomal sin presentar toxicidad renal.
Por presentar leucocitosis, se realizó una PAMO, que evidenció una segunda recaída medular, por lo que ingresó a Cuidados Paliativos Terminales y falleció por progresión de la enfermedad de base.

\section{DISCUSIÓN}

La sinusitis se define como la inflamación de la mucosa del seno paranasal. La rinosinusitis fúngica ocurre en formas agudas y crónicas, invasivas y no invasivas. La rinosinusitis invasiva ocurre de modo casi exclusivo en pacientes inmunosuprimidos, ${ }^{2}$ pero, en ocasiones, se presenta en personas previamente sanas. ${ }^{4}$

A menudo, comienza como una úlcera o escara negra indolora en el septo nasal o paladar y se extiende a través de la mucosa de los tejidos blandos y huesos yuxtapuestos. Clínicamente, el paciente se presenta con fiebre, tos, costras de mucosa nasal, epistaxis y cefalea. ${ }^{4}$

La diseminación hematógena y la angioinvasión son frecuentes. La destrucción ósea agresiva de las paredes del seno se produce rápidamente, y la diseminación intraorbitaria, intracraneal y maxilofacial es común. ${ }^{2}$

Los estudios histopatológicos muestran invasión de las hifas a los vasos sanguíneos, vasculitis con trombosis, hemorragia e infartos de tejidos, inflamación aguda neutrofílica ${ }^{2}$ (Figura 2).

La TAC sin contraste demuestra el compromiso del seno paranasal y la cavidad nasal implicados. La resonancia magnética es excelente para evaluar la extensión intracraneal e intraorbitaria de la enfermedad. ${ }^{2}$

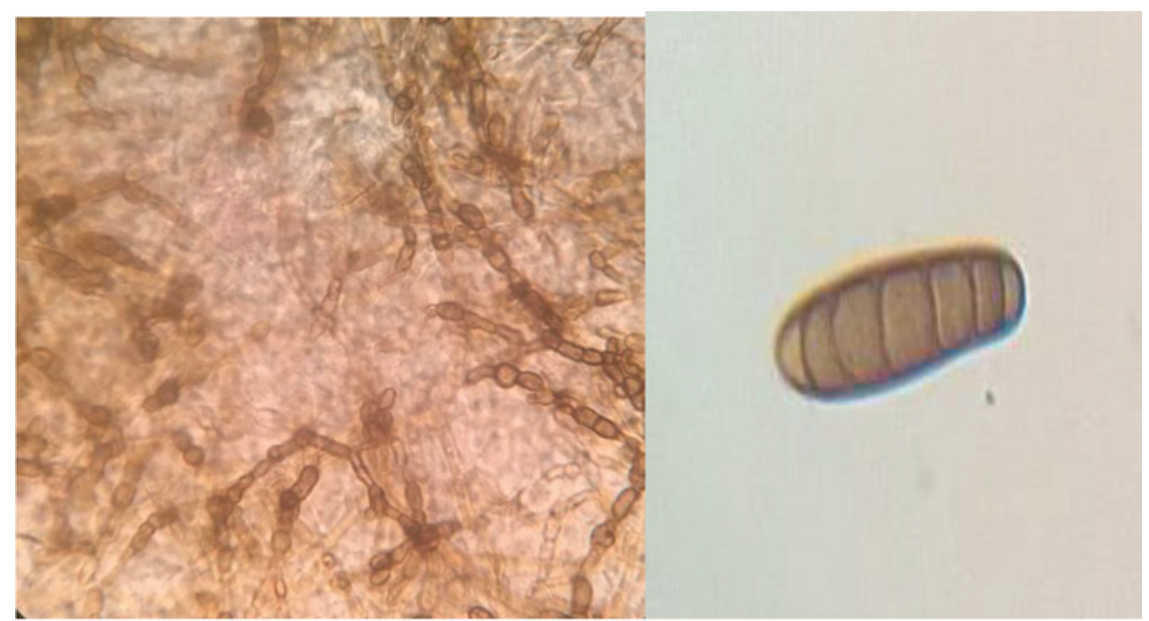

B. 
Los hongos saprofíticos, especialmente los del orden Mucorales, son agentes etiológicos habituales. ${ }^{4}$

El tratamiento implica un desbridamiento quirúrgico amplio y agresivo, anfotericina B intravenosa o preparación lipídica de anfotericina B y la corrección de la inmunosupresión subyacente, ${ }^{2}$ y se indica, cuando sea posible y en acuerdo con el hematooncológo, el uso de factores de estimulación de colonias de granulocitos o trasfusión de granulocitos.

Los pacientes que no se recuperan de su neutropenia tienen mal pronóstico independientemente de las medidas terapéuticas adyuvantes. ${ }^{2}$

Es la forma más letal de sinusitis fúngica, con una tasa de mortalidad de $50 \%$ a $80 \% .^{2}$ El género Exserohilum (familia Pleosporaceae, orden Pleosporales) son hongos filamentosos y dematiáceos caracterizados por conidios largos, multiseptados con hilio sobresaliente y la formación de colonias de color negro. Solo tres especies, E. rostratum, E. longirostratum y E. mcginnisii, son patógenos para los seres humanos. ${ }^{3}$ Son hongos ambientales comunes en la hierba y el suelo. ${ }^{3}$

La infección por estos agentes fúngicos es, en la mayoría de los casos, sistémica y afecta, principalmente, a los senos paranasales y los pulmones. Es más frecuente en pacientes inmunosuprimidos, aunque puede presentarse en pacientes inmunocompetentes. Exserohilum rostratum es el más frecuentemente aislado. ${ }^{3} \mathrm{La}$ infección es confirmada por análisis de laboratorio mediante cultivo e histopatología de biopsia y se basa en hallazgos morfológicos. El ensayo de reacción en cadena de la polimerasa (polymerase chain reaction; $\mathrm{PCR}$, por sus siglas en inglés) puede ser útil en la identificación del patógeno. ${ }^{3}$

En la literatura internacional, han sido publicados 19 casos de enfermedad por Exserohilum spp. en pacientes menores de 20 años, $^{3}$ de los cuales solo 9 eran pacientes inmunocomprometidos (Tabla 1)..$^{5-12}$

En los pacientes inmunocomprometidos, la localización más frecuente fue la rinosinusal sola o acompañada por compromiso de otros órganos, ${ }^{6-10}$ pero también se puede presentar como lesiones aisladas en la piel.5,11,12 El germen más frecuentemente aislado fue E. rostratum. . $-7,9,11,12$

Los factores predisponentes, en su mayoría, fueron leucemia linfoblástica y mieloblástica aguda. ${ }^{5,8,10,11}$ En cuanto al tratamiento antifúngico de elección, fue anfotericina B (desoxicolato o liposomal); $5-11$ en algunos casos, asociada a otros antifúngicos. ${ }^{5,6,8-10}$ En casi la mitad de los casos, además, se realizó el tratamiento quirúrgico. ${ }^{9-11}$ En cuanto al pronóstico, 5 de los 9 pacientes reportados en la literatura fallecieron; ${ }^{6-9,12}$ en la mayoría de los casos, se trató de enfermedad fúngica invasiva.

\section{CONCLUSIÓN}

Las infecciones fúngicas son cada vez más frecuentes en pacientes inmunosuprimidos, y los hongos pertenecientes al género Exserohilum son patógenos emergentes. Es importante sospechar la etiología fúngica en este tipo de pacientes para poder realizar un tratamiento precoz y adecuado, que implica la remoción quirúrgica del foco infeccioso, el tratamiento antifúngico y la resolución de la neutropenia.

TABLA 1. Casos de enfermedad invasiva por Exserohilum en pacientes pediátricos inmunosuprimidos publicados en la literatura

\begin{tabular}{|c|c|c|c|c|c|c|c|c|}
\hline $\begin{array}{l}\text { N. } \\
\text { de re } \\
\end{array}$ & $\begin{array}{l}\text { Autor } \\
\text { ef. }\end{array}$ & $\begin{array}{c}\text { Año de } \\
\text { publicación }\end{array}$ & $\begin{array}{c}\text { Agente } \\
\text { n etiológico } \\
\end{array}$ & $\begin{array}{l}\text { Edad/ } \\
\text { sexo }\end{array}$ & $\begin{array}{c}\text { Patología } \\
\text { de base }\end{array}$ & Localización & Tto. & Evolución \\
\hline 5 & Moneymaker et al. & 1986 & E. rostratum & 3 años $/ M$ & LLA & Piel & $\mathrm{AMB}, 5 \mathrm{FC}$ & Curación \\
\hline 6 & Sharkey et al. & 1990 & E. rostratum & 5 años / F & Aplasia & SPN, tej. blandos & AMB, ITR & Muerte \\
\hline 7 & Aquino et al. & 1994 & E. rostratum & 7 años / F & Aplasia & SPN, pulmón & AMB y GM-CSF & Muerte \\
\hline 8 & Levy et al. & 2003 & Exserohilum spp. & 8 años / M & LMA & Piel, SPN, pulmón & AMB, ITR & Muerte \\
\hline 9 & Lasala et al. & 2005 & E. rostratum & 18 años / F & Aplasia & SPN & Qx, AMB, vori, GM-CSF & Muerte \\
\hline 10 & Adler et al. & 2006 & Exserohilum spp. & 8 años / F & LLA & SPN, pulmón & Qx, AMB, vori & Curación \\
\hline 10 & Adler et al. & 2006 & Exserohilum spp. & 3 años / F & LLA & SPN & Qx, AMB, ITR & Curación \\
\hline 11 & Saint-Jean et al. & 2007 & E. rostratum & 3 años / M & LLA & Piel & Qx, AMB & Curación \\
\hline 12 & Juhas, E. et al. & 2012 & E. rostratum & 7 días / $\mathrm{M}$ & SAM & Piel & No & Muerte \\
\hline
\end{tabular}

N. ${ }^{o}$ de ref.: número de referencia; Tto.: tratamiento; LLA: leucemia linfoblástica aguda; LMA: leucemia mielioide aguda; SAM: síndrome de activación macrofágica; SPN: senos paranasales; AMB: anfotericina B liposomal; 5FC: 5 fluorcitosina; ITR: itraconazol; vori: voriconazol; Qx: cirugía; GM-CSF: factor estimulante de colonias de granulocitos. 


\section{REFERENCIAS}

1. Vallejo LlamasJC, Ruiz-CampsI. Infección fúngica invasora en los pacientes hematológicos. Enferm Infecc Microbiol Clin 2012; 30(9):572-9.

2. Apuhan T, Küçükbayrak A, Hakyemez I. Fungal sinusitis in immunocompromised hosts. Afr J Microbiol Res 2011; 5(13):1577-80.

3. Katragkou A, PanaZD, Kontoyiannis DP, etal. Exserohilum infections: Review of 48 cases before the 2012 United States outbreak. Med Mycol 2014; 52(4):376-86.

4. Chakrabarti A, Das A, Panda N. Overview of fungal rhinosinusitis. Indian J Otolaryngol Head Neck Surg 2004; 56(4):251-8.

5. Moneymaker CS, Shenep JL, Pearson TA, et al. Primary cutaneous phaeohyphomycosis due to Exserohilum rostratum (Drechslera rostrata) in a child with leukemia. Pediatr Infect Dis 1986; 5(3):380-2.

6. Sharkey PK, Graybill JR, Rinaldi MG, et al. Itraconazole treatment of phaeohyphomycosis. J Am Acad Dermatol 1990; 23(3 Pt 2):577-86.
7. Aquino VM, Norvell JM, Krisher K, etal. Fatal disseminated infection due to Exserohilum rostratum in a patient with aplastic anemia: case report and review. Clin Infect Dis 1995; 20(1):176-8.

8. Levy I, Stein J, Ashkenazi S, et al. Ecthyma gangrenosum caused by disseminated Exserohilum in a child with leukemia: a case report and review of the literature. PediatrDermatol 2003; 20(6):495-7.

9. Lasala PR, Smith MB, McGinnis MR, et al. Invasive Exserohilum sinusitis in a patient with aplastic anemia. Pediatr Infect Dis J 2005; 24(10):939-41.

10. Adler A, Yaniv I, Samra Z, et al. Exserohilum: an emerging human pathogen. Eur J Clin Microbiol Infect Dis 2006; 25(4):247-53.

11. Saint-Jean M, St-Germain G, Laferrière $C$, et al. Hospital acquired phaeohyphomycosis due to Exserohilum rostratum in a child with leukemia. Can J Infect Dis Med Microbiol 2007; 18(3):200-2.

12. Juhas E, Reyes-Mugica M, Michaels MG, et al. Exserohilum infection in an immunocompromised neonate. Pediatr Dermatol 2012; 30(6):e232-3. 